\title{
DIRECT PRODUCTS AND THE HOPF PROPERTY
}

\author{
Dedicated to the memory of Hanna Neumann
}

J. M. TYRER JONES

(Received 20 September 1972)

Communicated by M. F. Newman

\section{Introduction}

In [1] Neumann and Dey prove that the free product of two finitely generated Hopf groups is Hopf and ask whether a similar result holds for direct products. It is the purpose of this paper to show that this is not the case. We prove

THEOREM A. There exists a finitely generated group $G$ satisfying the following conditions:

(i) $G$ is isomorphic to a proper direct factor of itself;

(ii) $G$ is the direct product of two Hopf groups.

The method of proof can easily be adapted to give the following result:

THEOREM B. There exists a non-trivial finitely generated group $G_{0}$ isomorphic to its own direct square.

Theorem B provides an answer to a question raised by $J$. Wiegold and $\mathrm{K}$. W. Gruenberg in lectures, for $G_{0}$ is a finitely generated group having the property that there is a bound on the number of generators of the direct power $G_{0}^{n}$ as $n$ varies through the natural numbers.

The construction of $G$ proceeds as follows: We first select a suitable member $S$ of the family of simple groups constructed by Camm in [2], and form the cartesian power $C=S^{N}$ where $N$ denotes the natural numbers. It is then possible to choose a finitely generated subgroup $G$ of $C$ in such a way that conditions (i) and (ii) of Theorem A are satisfied. The proof of (ii) is very long, so we merely sketch the method of proof here, referring the reader to [3] for full details.

The construction of $G_{0}$ is very similar to that of $G$, except that, in this case we use an HNN extension of one of Camm's groups as the 'base group' $S_{0}$. 
It should be noted, however, that it is possible to use various other groups in place of $S_{0}$ : in particular, the description of the 'base group' may be considerably simplified at the expense of increasing the number of generators of $G_{0}$. The possibility of such a simplification was pointed out to me by Miller, and his example is to be found in [3].

I should like to thank Dr. P. M. Neumann, under whose supervision this work was carried out, and Dr. C. F. Miller III, with whom I had many valuable discussions, for their help and advice. I am also indebted to the Science Research Council for their financial support.

\section{The proof of Theorem $A$}

We begin with a brief description of Camm's simple groups. Let $D=\langle a, p\rangle$ and $E=\langle b, q\rangle$ be two two-generator free groups. Let $I$ be the set $\{ \pm 1, \pm 2, \pm 3, \cdots\}$ and let $\rho, \sigma$ and $\tau$ be permutations of $I$. We put $g_{i}=a^{i} p^{\rho(i)}$ and $h_{i}=b^{i} q^{\sigma(i)}$ for each $i \in I$. The elements $g_{i}$ then freely generate a subgroup $U$ of $D$, and the elements $h_{i}$ freely generate an isomorphic subgroup $V$ of $E$. We form the free product $S_{\rho \cdot \sigma \cdot \tau}$ of $D$ and $E$ amalgamating $U$ with $V$ under the isomorphism $\phi$ defined by

$$
\phi\left(g_{i}\right)=h_{\tau(i)} \quad \text { for each } i \in I .
$$

In [2] Camm shows that for suitable choices of $\rho, \sigma$ and $\tau$ the groups $S_{\rho, \sigma, \tau}$ are all simple. She does not define $\rho, \sigma$ and $\tau$ completely. The definitions she stipulates all map positive integers to positive integers, and other definitions can be made arbitrarily, subject to only one condition, which states essentially that $|\rho(n)|$, $|\sigma(n)|$ and $|\tau(n)|$ are 'not much bigger' than $|n|$. It is easy to satisfy this condition by defining $\rho, \sigma$ and $\tau$ on the negative integers in such a way that each of them fixes each block $\{-20 r,-20 r+1,-20 r+2, \cdots,-20 r+19\}(r \in N)$ set-wise.

We now construct our 'base group' $S$. This is to be a group $S_{\rho \sigma, \tau}$ with $\rho, \sigma$ and $\tau$ defined in the following way:

(I) $\rho, \sigma$ and $\tau$ are defined as in [2] wherever these definitions are made. In particular

$$
\rho(1)=\sigma(1)=\tau(1)=1 \text {. }
$$

(II) The definitions of $\rho, \sigma$ and $\tau$ on the positive integers are not completely determined by (I). We make further definitions as follows:

$$
\begin{aligned}
& \rho(11)=\sigma(11)=\tau(11)=11 \\
& \rho(12)=\sigma(12)=\tau(12)=12 \\
& \rho(13)=\tau(13)=13, \sigma(13)=14 .
\end{aligned}
$$

All other definitions on the positive integers are made arbitrarily, subject to the condition stipulated in [2]. 
(III) The following definitions are made on the negative integers:

Let

$$
\begin{aligned}
& P_{1}=\left\{2^{r}: r>0, r \in Z\right\}, \\
& P_{2}=N \backslash P_{1}, \quad \text { and let } \\
& \Delta_{t}=\{n \in Z:-20 t \leqq n \leqq-20 t+19\} .
\end{aligned}
$$

For each $t \in P_{2}, \rho, \sigma$ and $\tau$ fix $\Delta_{t}$ point-wise. For each $t \in P_{1}, \rho, \sigma$ and $\tau$ fix $\Delta_{t}$ set-wise, and induce on $\Delta_{t}$ permutations $\rho_{t}, \sigma_{t}$ and $\tau_{t}$ defined (in the usual notation, as products of disjoint cycles) by

$$
\begin{aligned}
& \rho_{t}=(-20 t+9,-20 t+10), \\
& \sigma_{t}=(-20 t,-20 t+7,-20 t+4,-20 t+3,-20 t+1) \\
& \quad \cdot(-20 t+2,-20 t+5,-20 t+6), \\
& \tau_{t}=(-20 t+2,-20 t+3) .
\end{aligned}
$$

Let $C$ be the cartesian power $S^{N}$, and let $D$ be the subgroup of $C$ consisting of all functions of finite support. An element $f$ of $C$ will be written as an infinite vector $\left(f_{1}, f_{2}, f_{3}, \cdots\right)$, where the $i$ th entry $f_{i}$ denotes the value of $f$ at $i$. Then $G$ is the subgroup of $C$ generated by

$$
\begin{aligned}
& x=\left(b^{-1} a, b^{-1} a, b^{-1} a, \cdots\right) \\
& y=(p, p, p, \cdots) \\
& z=\left(p^{20} a p^{-19}, p^{40} a p^{-39}, p^{60} a p^{-59}, \cdots\right) .
\end{aligned}
$$

We define $S_{i}$ to be the subgroup $\{f \in C: f(j)=1$ for all $j \neq i\}$ of $C$, so that $S_{i}$ is isomorphic to $S$ for each $i \in N$.

Proposition 1. $G \cong S \times G .1$

PROof. We first prove that $G$ intersects $S_{1}$ non-trivially. Thus we wish to find some element $w \neq 1$ of $S$ such that

$$
(w, 1,1,1, \cdots, 1, \cdots) \in G .
$$

We prove that the element

$$
w=\left(y^{-31} z y^{31}\right)^{-1} x\left(y^{-31} z y^{31}\right) x^{-1}
$$

of $G$ has this form.

The value of $w$ at $t \in N$ is

$$
\begin{aligned}
& \left(p^{20 t-31} a p^{-20 t+32}\right)^{-1}\left(b^{-1} a\right)\left(p^{20 t-31} a p^{-20 t+32}\right)\left(b^{-1} a\right)^{-1} \\
& =\left(p^{20(t-1)-11} a p^{-20(t-1)+12}\right)^{-1}\left(b^{-1} a\right)\left(p^{20(t-1)-11} a p^{-20(:-1)+12}\right) \cdot \\
& \cdot\left(b^{-1} a\right)^{-1} .
\end{aligned}
$$


Now by definitions (III), $\rho, \sigma$ and $\tau$ each fix $-20 t+11,-20 t+12$ and $-20 t+13$ for each $t>0$. Also, by definitions (I), $\rho, \sigma$ and $\tau$ each fix 1 , so for $t>1$,

$$
\begin{aligned}
& \left(b^{-1} a\right)\left(p^{20(t-1)-11} a p^{-20(t-1)+12}\right)\left(b^{-1} a\right)^{-1} \\
& =b^{-1}(a p)\left(a^{-20(t-1)+12} p^{-20(t-1)+12}\right)^{-1}\left(a^{-20(t-1)+13} p^{-20(t-1)+13}\right)\left(p^{-1} a^{-1}\right) b \\
& =q\left(b^{-20(t-1)+12} q^{-20(t-1)+12}\right)^{-1}\left(b^{-20(t-1)+13} q^{-20(t-1)+13}\right) q^{-1} \\
& =q^{20(t-1)-11} b q^{-20(t-1)+12} \\
& =\left(b^{-20(t-1)+11} q^{-20(t-1)+11}\right)^{-1}\left(b^{-20(t-1)+12} q^{-20(t-1)+12}\right) \\
& =\left(a^{-20(t-1)+11} p^{-20(t-1)+11}\right)^{-1}\left(a^{-20(t-1)+12} p^{-20(t-1)+12}\right) \\
& =p^{20(t-1)-11} a p^{-20(t-1)+12} .
\end{aligned}
$$

Thus the value of $w$ at $t$ is 1 for all $t>1$. But the value of $w$ at 1 is

$$
\left(p^{-11} a p^{12}\right)^{-1}\left(b^{-1} a\right)\left(p^{-11} a p^{12}\right)\left(b^{-1} a\right)^{-1},
$$

and, by definitions (II),

$$
\begin{aligned}
& \left(b^{-1} a\right)\left(p^{-11} a p^{12}\right)\left(b^{-1} a\right)^{-1}=b^{-1}(a p)\left(a^{12} p^{12}\right)^{-1}\left(a^{13} p^{13}\right)\left(p^{-1} a^{-1}\right) b \\
& \quad=q\left(b^{12} q^{12}\right)^{-1}\left(b^{13} q^{14}\right) q^{-1} \\
& \quad=q^{-11} b q^{13}=\left(b^{11} q^{11}\right)^{-1}\left(b^{12} q^{12}\right) q=\left(a^{11} p^{11}\right)^{-1}\left(a^{12} p^{12}\right) q=p^{-11} a p^{12} q .
\end{aligned}
$$

Thus the value of $w$ at 1 is $q$, and consequently $G$ intersects $S_{1}$. But $S$ is simple, and the set $\{f(1): f \in G\}$ is the whole of $S$. Thus $G \cap S_{1}=S_{1}$, showing that $S_{1}$ is a direct factor of $G$. Let $\alpha: C \rightarrow C$ be the monomorphism given by

$$
(f \alpha)(i)= \begin{cases}f(i-1) & \text { for } i>1 \\ 1 & \text { for } i=1\end{cases}
$$

and let $G_{1}=G \alpha$. Then $G_{1}$ is generated by

$$
\begin{aligned}
& x_{1}=\left(1, b^{-1} a, b^{-1} a, b^{-1} a, \cdots\right) \\
& y_{1}=(1, p, p, p, \cdots) \\
& z_{1}=\left(1, p^{20} a p^{-19}, p^{40} a p^{-39}, p^{60} a p^{-59}, \cdots\right)
\end{aligned}
$$

and is clearly a direct complement for $S_{1}$ in $G$. Moreover $\left.\alpha\right|_{G}$ is an isomorphism from $G$ onto $G_{1}$. Consequently $G \cong S \times G$, as required.

Let $C_{1}$ and $C_{2}$ denote the cartesian powers $S^{P_{1}}$ and $S^{P_{2}}$, regarded in the obvious way as subgroups of $C$. For each $f \in G$ we define functions $f_{A}, f_{B} \in C$ as follows:

$$
f_{A}(i)= \begin{cases}f(i) & \text { if } i \in P_{1} \\ 1 & \text { otherwise }\end{cases}
$$




$$
f_{B}(i)= \begin{cases}f(i) & \text { if } i \in P_{2} \\ 1 & \text { otherwise. }\end{cases}
$$

Let $A$ and $B$ denote the subgroups $\left\{f_{A}: f \in G\right\}$ and $\left\{f_{B}: f \in G\right\}$ of $C$ respectively. In the usual notation we denote a commutator $a^{-1} b^{-1} a b$ by $[a, b]$.

\section{Proposition 2. $G=A \times B$.}

Proof. We need only show that $A \leqq G$. Using definitions (III), we see that the following relations hold in $S$ :

$$
\begin{array}{ll}
\left(b^{-1} a\right)\left(p^{20 t} a p^{-20 t+1}\right)\left(a^{-1} b\right)=p^{20 t} a p^{-20 t+1} & \text { if } t \in P_{2} \text {, and } \\
\left(b^{-1} a\right)\left(p^{20 t} a p^{-20 t+1}\right)\left(a^{-1} b\right)=p^{20 t+1} a^{2} p^{-20 t+1} & \text { if } t \in P_{1} .
\end{array}
$$

Consequently

$$
\begin{aligned}
{\left[x^{-1}, z^{-1}\right] } & =\left(p^{21} a p^{-20}, p^{41} a p^{-40}, 1, p^{81} a p^{-80}, 1,1, \cdots\right) \\
& =y z_{A} y^{-1} \quad \text { (where } z_{A} \text { is defined as above), }
\end{aligned}
$$

which shows that $z_{A}$ and all its conjugates by powers of $y$ lie in $G$. But we also have

$$
\left(b^{-1} a\right)\left(p^{20 t-3} a p^{-20 t+4}\right)\left(a^{-1} b\right)=p^{20 t-6} a p^{-20 t+7} q \quad \text { for all } t \in P_{1},
$$

and so

$$
x\left(y^{-3} z_{A} y^{3}\right) x^{-1}=\left(y^{-6} z_{A} y^{6}\right)\left(x_{A} y_{A}\right),
$$

which shows that $x_{A} y_{A}$ lies in $G$. Further

$$
\left(b^{-1} a\right)\left(p^{20 t-10} a p^{-20 t+11}\right)\left(a^{-1} b\right)=p^{20 t-9} a p^{-20 t+11} \quad \text { for all } t \in P_{1},
$$

and so

$$
x\left(y^{-10} z_{A} y^{10}\right) x^{-1}=\left(y^{-9} z_{A} y^{9}\right) y_{A},
$$

which shows that $y_{A}$ lies in $G$. Thus $A=\left\langle x_{A}, y_{A}, z_{A}\right\rangle \leqq G$, and the result follows.

By Proposition 1, $G$ is non-Hopf, and by Proposition $2, G=A \times B$. Thus we need only show that $A$ and $B$ are Hopf. We give sketches of the proofs, referring the reader to [3] for details.

Proposition 3. A is Hopf.

Proof. We must prove that every ependomorphism of $A$ is an automorphism. To this end, we factor out a characteristic subgroup of $A$, and look at the ependomorphisms of the corresponding factor group.

Let $D_{1}$ be the subgroup of $C_{1}$ consisting of all functions of finite support. An easy extension of the proof of Proposition 1 shows that $D_{1} \leqq A$. In fact $D_{1}$ is the direct product of the minimal normal subgroups of $A$, for it is easily seen that any normal subgroup $N$ of $A$ intersects all those $S_{1}\left(i \in P_{1}\right)$ for which there 
is some $n \in N$ such that $n(i) \neq 1$. Consequently, for any ependomorphism $\phi$ of $A$ we have $\phi\left(D_{1}\right) \leqq D_{1}$. We investigate the ependomorphisms of the factor group $\bar{A}=A / D_{1}$, denoting the images of $x_{A}, y_{A}$ and $z_{A}$ in $\bar{A}$ by $x, y$ and $z$. We put $x y=t, y^{3} z y^{-3}=u$, and prove

Lemma 3.1. $L=\langle y, u\rangle$ and $M=\langle t, u\rangle$ are both two-generator free groups. Let

$$
\begin{gathered}
H=\left\langle y^{-k} u y^{k}(k \in Z, k \neq 11,12,13) y^{-11} u y^{12}, y^{-12} u y^{13}, y^{-13} u y^{11}\right\rangle \leqq L, \\
K=\left\langle t^{-k} u t^{k}(k \in Z, k \notin\{2,3,4, \cdots, 10\}), t^{-2} u t^{9}, t^{-3} u t^{7}, t^{-4} u t^{5},\right. \\
\left.t^{-5} u t^{6}, t^{-6} u t^{8}, t^{-7} u t^{10}, t^{-8} u t^{3}, t^{-9} u t^{4}, t^{-10} u t^{2}\right\rangle \leqq M,
\end{gathered}
$$

and let $\theta$ be the map from $H$ to $K$ defined by

$$
\begin{array}{rlrl}
\theta\left(y^{-k} u y^{k}\right) & =t^{-k} u t^{k} & \text { for all } k \geqq 14, \text { and for all } k \leqq 1, \\
\theta\left(y^{-2} u y^{2}\right) & =t^{-2} u t^{9}, & \theta\left(y^{-3} u y^{3}\right) & =t^{-10} u t^{2}, \quad \theta\left(y^{-4} u y^{4}\right)=t^{-3} u t^{-1} u t^{3}, \\
\theta\left(y^{-5} u y^{5}\right) & =t^{-3} u^{-1} t^{8}, \quad \theta\left(y^{-6} u y^{6}\right)=t^{-8} u t^{-1} u t^{5}, \theta\left(y^{-7} u y^{7}\right)=t^{-6} u t^{8}, \\
\theta\left(y^{-8} u y^{8}\right) & =t^{-9} u t^{5}, \quad \theta\left(y^{-9} u y^{9}\right)=t^{-5} u t^{6}, \quad \theta\left(y^{-10} u y^{10}\right)=t^{-7} u t^{10}, \\
\theta\left(y^{-11} u y^{12}\right) & =t^{-11} u t^{11}, \theta\left(y^{-12} u y^{13}\right)=t^{-13} u t^{13}, \theta\left(y^{-13} u y^{11}\right) & =t^{-12} u t^{12} .
\end{array}
$$

Then $\theta$ is an isomorphism, and $\bar{A}=*(L, M ; H, K, \theta)$, the free product of $L$ und $M$ amalgamating $H$ with $K$ under the isomorphism $\theta$.

Proof. A relation $w(x, y, z)=1$ holds in $\bar{A}$ if and only if the corresponding word $w_{A}=w\left(\boldsymbol{x}_{A}, \boldsymbol{y}_{A}, z_{A}\right)$ of $A$ lies in $D_{1}$, that is, if and only if the value $w_{A}(i)$ $=w\left(b^{-1} a, p, p^{20 i} a p^{-20 i+1}\right)$ of $w_{A}$ at $i$ is trivial for all but finitely many integers $i \in P_{1}$. Thus we may find relations in $\bar{A}$ by making calculations within $S$.

As the subgroups $\left\langle p, p^{20 i+3} a p^{-20 i-2}\right\rangle$ and $\left\langle q, q^{20 i+3} b q^{-20 i-2}\right\rangle$ of $S$ are free for each $i \in P_{1}$, the above reasoning shows that the subgroups $\langle y, u\rangle$ and $\langle t, u\rangle$ of $\bar{A}$ are free. It follows from cancellation arguments in the free groups $L$ and $M$ that $H$ and $K$ are free on the stated generators, so that $\theta$ is indeed an isomorphism. Moreover the elements $\left\{y^{i}: i \in Z\right\}$ and $\left\{t^{i}: i \in Z\right\}$ form complete sets of coset representatives for $H$ in $L$ and $K$ in $M$ respectively. (An argument similar to that required here may be found in [2], Lemma 3.) Using definitions (III), we may check that the relations determined by $\theta$ all hold in $\bar{A}$, so that $\bar{A}$ is a homomorphic image of $R=*(L, M ; H, K, \theta)$.

If $\bar{A}$ were a proper homomorphic image of $R$, there would be an element $w$ of normal form length greater than one in $R$, such that $w$ has trivial image in $\bar{A}$. Let $w$ be a word of length $n$ in $R$, say $w=h s_{1} s_{2} \cdots s_{n}$ where $h \in H$ and for each index $i$ the factor $s_{i}$ is a power of $y$ or of $t$, taken alternately. We note that in $S$ the powers of $p$ form a transversal for $U$ in $D$ and the powers of $q$ form a transversal for $V$ in $E$. We note further that for each generator $g$ of $H$ the value of $g_{A}$ 
at $i$ lies in the amalgamated subgroup $U$ of $S$ for all but finitely many $i \in P_{1}$. Now $h$ is a word involving only finitely many of the generators of $H$, so it follows that the value of $h_{A}$ at $i$ lies in the amalgamated subgroup $U$ of $S$ for all but finitely many $i \in P_{1}$. Moreover the values of $y_{A}$ and $t_{A}$ at $i$ are $p$ and $q$ respectively. Consequently, for all but finitely many $i \in P_{1}$, the value of $w_{A}$ at $i$ is reduced as written and has normal form length $n$ in $S$, so that the image of $w$ in $A$ is not equal to 1 . Thus $\bar{A}=R$, as required.

Having determined the structure of $\bar{A}$, we are now in a position to find its ependomorphisms. We shall need the following two theorems, both to be found in [4] (Theorem 4.5, p. 209 and Corollary N4, p. 169 respectively).

THEOREM 1. Let $G=*(A, B ; H, K, \phi)$, and suppose that $x, y \in G$ are such that $x y=y x$. Then either

(i) $x$ or $y$ is in a conjugate of $H$, or

(ii) if neither $x$ nor $y$ is in a conjugate of $H$, but $x$ is in a conjugate of $a$ factor, then $y$ is in that same conjugate of a factor, or

(iii) if neither $x$ nor $y$ is in a conjugate of a factor, then $x=g \mathrm{gg}^{-1} \cdot W^{j}$ and $y=g h^{\prime} g^{-1} \cdot W^{k}$, where $g, W \in G, h, h^{\prime} \in H$ and $g h g^{-1}, g h^{\prime} g^{-1}$ and $W$ commute in pairs.

THEOREM 2. Let Aut $(G)$ be the automorphism group of a group $G$, and let Inn $(G)$ be the normal subgroup of Aut $(G)$ consisting of inner automorphisms. Let $F_{2}$ denote a free group of rank two and $A_{2}$ a free abelian group of rank two. Then

$$
\frac{\operatorname{Aut}\left(F_{2}\right)}{\operatorname{Inn}\left(F_{2}\right)} \cong \operatorname{Aut}\left(A_{2}\right) \text {. }
$$

Moreover Aut $\left(A_{2}\right)$ is isomorphic to $G L(2, Z)$, that is, the multiplicative group of $2 \times 2$ matrices with integer entries, and determinant \pm 1 .

We now sketch a proof of

LEMMA 3.2. Every ependomorphism of $\bar{A}$ is an inner automorphism.

Proof. Let $\phi$ be an ependomorphism of $\bar{A}$. Using the relations given in Lemma 3.1, the words $x\left(y^{i} z y^{-i}\right) x^{-1}$ of $\bar{A}$ may be evaluated for each $i \in Z$. We find that $x$ commutes with $y^{i} z y^{-i}$ if and only if $i \geqq 3$ or $i \leqq-11$. Thus $\phi(x)$ commutes with $\phi\left(y^{i} z y^{-i}\right)$ for these values of $i$, and we may apply Theorem 1 to establish the normal form structure of the images under $\phi$ of elements of $\bar{A}$. Without loss of generality we may assume that $\phi(x)$ is cyclically reduced. Suppose first that $\phi(x)$ lies in a factor, but not in a conjugate of $H$. By Theorem $1, \phi\left(y^{3} z y^{-3}\right)$ and $\phi\left(y^{4} z y^{-4}\right)$ both lie in that same factor or in a conjugate of $H$. A length argument shows that even in the latter case they must both lie in the same factor as $\phi(x)$. Now the factors are free, and so it follows that $\phi(x), \phi\left(y^{3} z y^{-3}\right)$ and $\phi\left(y^{4} z y^{-4}\right)$ are all powers of the same element. Thus, in particular, 


$$
\phi\left(\left[y^{3} z y^{-3}, y^{4} z y^{-4}\right]\right)=1 \text {. }
$$

We may conjugate this relation by powers of $\phi(y)$ and by $\phi(x)$. Using the fact that $\phi(x)$ commutes with $\phi\left(y^{i} z y^{-i}\right)$ for all $i \geqq 3$ or $\leqq-11$, and using the relation

$$
x\left(y^{-10} z y^{10}\right) x^{-1}=y^{-9} z y^{10}
$$

which holds in $A$, we may now deduce that $\phi(y)=1$. Having established this, it is not difficult to deduce from the relations of $\bar{A}$ that $\phi(z)=1$ also, and consequently that $\phi$ is not an ependomorphism. A similar argument deals with the case where $\phi\left(y^{3} z y^{-3}\right)$ lies in a conjugate of a factor, but not in a conjugate of $H$.

We next consider the case when both $\phi(x)$ and $\phi\left(y^{3} z y^{-3}\right)$ lie in (possibly different) conjugates of $H$. This case can also be eliminated. The argument is basically the same as that used in the above case, but the proof is longer and the normal form theorem, with associated length and cancellation arguments, is used more strongly. We refer the reader to [3] for details.

Next we assume that $\phi\left(y^{3} z y^{-3}\right)$ lies in a conjugate of $H$. Without loss of generality we may assume that $\phi(x)$ is cyclically reduced. The above results show that $\phi(x)$ cannot lie in a factor, so $\phi(x)$ has normal form length greater than one. An easy length argument then shows that $\phi\left(y^{i} z y^{-i}\right)$ lies in $H$ for all $i \geqq 3$ or $\leqq-11$. The next step is to establish the structure of $\phi(y)$. It is easy to eliminate the case in which $\phi(y) \in H$ : if this were true, we would have $\phi\left(y^{i} z y^{-i}\right) \in H$ for each $i \in Z$, and then applying $\phi$ to the relation

$$
x\left(y^{-3} z y^{3}\right) x^{-1}=y^{-6} z y^{6}(x y)
$$

of $A$ would yield an equation in which the right-hand side is cyclically reduced of length greater than one, but in which the left-hand side is not cyclically reduced unless it lies in a factor. A similar argument eliminates the case where $\phi(y)$ does not lie in a conjugate of a factor, and yet another argument of the same nature shows that we may assume that $\phi(y)$ lies within a factor. (A conjugation may be necessary to establish this, but it does not affect the fact that $\phi(x)$ is cyclically reduced.)

The proof continues in a similar manner (see [3]) until we obtain the following situation:

There exists an element $h \in H$ and elements $c$ and $d$ belonging to different factors $F(c), F(d)$ of $\bar{A}$ such that

$$
\begin{aligned}
\phi\left(y^{3} z y^{-3}\right) & =h \\
\phi(y) & =c \\
\phi(x) & =d c^{-1}
\end{aligned}
$$

and such that $h$ and $c$ together generate $F(c)$ and $h$ and $d$ together generate $F(d)$. 
We consider first the case in which $F(c)=L$ and $F(d)=M$. Now $h$ is equal to a word $w$ in the generators of $H$. Since $h$ and $c$ generate $L, h$ is a primitive element of the two-generator free group $L$, so we may apply Theorem 2. This shows that the sum of the exponents of $y^{3} z y^{-3}$ and the sum of the exponents of $y$ appearing in $w$ must be coprime. Suppose that the total sum of the exponents of all generators $y^{i}\left(y^{3} z y^{-3}\right) y^{-i}(i \neq-11,-12,-13)$ appearing in $w$ is $\alpha_{0}$, and suppose that $y^{-11}\left(y^{3} z y^{-3}\right) y^{12}$ appears to total exponent $\alpha_{1}$, that $y^{-12}\left(y^{3} z y^{-3}\right) y^{13}$ appears to total exponent $\alpha_{2}$ and that $y^{-13}\left(y^{3} z y^{-3}\right) y^{11}$ appears to total exponent $\alpha_{3}$. Suppose further that $\alpha_{1}+\alpha_{2}-2 \alpha_{3} \neq 0$. Now $h$ and $c$ generate $L$, and $c^{-i} h c^{i} \in H$ for all but finitely many $i \in Z$. It is not difficult to deduce from this information that $y^{-1} h y^{i} \in H$ for all but finitely many $i \in Z$. But this cannot be the case if $\alpha_{1}+\alpha_{2}-2 \alpha_{3} \neq 0$. Thus $\alpha_{1}+\alpha_{2}-2 \alpha_{3}=0$, so applying Theorem 2 , we see that $\alpha_{0}+\alpha_{1}+\alpha_{2}+\alpha_{3}= \pm 1$. We now use the full strength of Theorem 2 , in the form

$$
\frac{\operatorname{Aut}\left(F_{2}\right)}{\operatorname{Inn}\left(F_{2}\right)} \cong \operatorname{Aut}\left(A_{2}\right) \cong G L(2, Z) \text {. }
$$

Since $L$ is a free group of rank two we may deduce that there exists an element $e$ of $L$ and integers $r, \varepsilon_{1}= \pm 1$, and $\varepsilon_{2}= \pm 1$ such that

$$
\begin{aligned}
\phi\left(y^{3} z y^{-3}\right) & =e\left(y^{3} z y^{-3}\right)^{e_{1}} e^{-1} \\
\phi(y) & =e\left(y^{\varepsilon}\left(y^{3} z y^{-3}\right)\right)^{-1} .
\end{aligned}
$$

But $e=h^{\prime} y^{j}$ for some $h^{\prime} \in H$ and some integer $j$. Thus, modulo an inner automorphism by $h^{\prime}$, we have

$$
\begin{aligned}
\phi\left(y^{3} z y^{-3}\right) & =\left(y^{3+j} z y^{-(3+j)}\right)^{\varepsilon_{1}} \in H \\
\phi(y) & =y^{\varepsilon_{2}}\left(y^{3+j} z y^{-(3+j)}\right)^{r}
\end{aligned}
$$

Now $\phi\left(y^{3} z y^{-3}\right)$ and $\phi(x y)$ together generate $M$, and so, by a further application of Theorem 2, we obtain

$$
\phi(x y)=\left(y^{3+j} z y^{-(3+j)}\right)^{l}(x y)^{\varepsilon_{3}}\left(y^{3+j} z y^{-(3+j)}\right)^{m}
$$

for some integers $l, m$ and $\varepsilon_{3}= \pm 1$.

Having found specific values for $\phi(x), \phi(y)$ and $\phi(z)$, we may now check through the defining relations of $\bar{A}$ to see whether any of the above maps are, in fact, ependomorphisms of $A$. We discover that the only case in which $\phi$ is an ependomorphism of $A$ is that in which $\varepsilon_{1}=\varepsilon_{2}=\varepsilon_{3}=1$ and $j=r=l$ $=m=0$. Consequently, modulo an inner automorphism, $\phi$ is the identity map. It follows that $\phi$ is an inner automorphism of $\bar{A}$. The case $F(c)=M, F(d)=L$ can be dealt with similarly, and eliminated.

The only remaining case is that in which $\phi\left(y^{3} z y^{-3}\right)$ has cyclically reduced length greater than one. This case may also be eliminated. The proof is long, but 
very similar to that for the case in which $\phi\left(y^{3} z y^{-3}\right)$ lies in a conjugate of $H$. We refer the reader to [3] for details. This completes the proof of Lemma 3.2.

We are now in a position to complete the proof of Proposition 3. Let $\phi$ be an ependomorphism of $A$. As noted at the beginning of the proof of Proposition 3, we have $\phi\left(D_{1}\right) \leqq D_{1}$ and so $\phi$ induces on $\bar{A}$ an ependomorphism, which, by Lemma 3.2, is an inner automorphism. Consequently there exist an element $g$ of $A$, and elements $\xi, \eta$ and $\zeta$ of $D_{1}$ such that

$$
\begin{aligned}
& \phi\left(x_{A}\right)=g x_{A} g^{-1} \xi \\
& \phi\left(y_{A}\right)=g y_{A} g^{-1} \eta \\
& \phi\left(z_{A}\right)=g z_{A} g^{-1} \zeta .
\end{aligned}
$$

We choose an integer $r$ such that $\xi, \eta$ and $\zeta$ all lie in $T=\left\{f \in D_{1}: f(i)=1\right.$ for all $\left.i>2^{r}\right\}$. It is now clear that for any $n>r$, we have $\phi\left(S_{2 n}\right)=S_{2 n}$, and that $\operatorname{ker}(\phi) \leqq T$. Further $\phi$ maps $D_{1}$ onto $D_{1}$ and $T$ onto $T$. But $T$ is a direct product of finitely many simple groups and, as such, is Hopf. Consequently $\operatorname{ker}(\phi)$ is trivial, so that $\phi$ is an automorphism of $A$. Thus $A$ is Hopf, as required.

We now turn our attention to the group $B$. We recall the definition of $x_{B}, y_{B}$ and $z_{B}$ made just before the statement of Proposition 2 .

Proposition 4. B is Hopf.

Proof. Let $D_{2}$ be the subgroup of $C_{2}$ consisting of all functions of finite support. Using the method of Proposition 3, we see that $B$ contains $D_{2}$ and that $D_{2}$ is the direct product of the minimal normal subgroups of $B$. We put $\bar{B}=B / D_{2}$ and consider the structure of $\vec{B}$.

Let $\bar{C}$ be the cartesian power $\bar{C}=\bar{A}^{N}$, and let $\bar{D}$ be the subgroup of $\bar{C}$ consisting of all functions of finite support. Let $T$ be the subgroup of $\bar{C}$ generated by

We prove

$$
\begin{aligned}
& X=(x, x, x, \cdots) \\
& Y=(y, y, y, \cdots) \\
& Z=\left(y^{20} z y^{-20}, y^{40} z y^{-40}, y^{60} z y^{-60}, \cdots\right) .
\end{aligned}
$$

LemMa 4.1. T contains $\vec{D}$. Further the map $\omega$ from $B$ to $\bar{C}$ defined by

$$
\begin{aligned}
& \omega\left(\boldsymbol{x}_{B}\right)=\boldsymbol{X} \\
& \omega\left(\boldsymbol{y}_{B}\right)=\mathbf{Y} \\
& \omega\left(\boldsymbol{z}_{\boldsymbol{B}}\right)=\boldsymbol{Z}
\end{aligned}
$$

is a homomorphism of $B$ onto $T$ with kernel $D_{2}$. (Consequently $\bar{B}$ is isomorphic to $T$, and may be identified with it under $\omega$.) 
Proor. Consideration of the elements $\left[X^{-1},\left(Y^{-20 t} Z Y^{20 t}\right)^{-1}\right](t \in N)$ of $T$ shows, by an argument similar to that used in the proof of Proposition 2, that $\bar{D} \leqq T$. Let $\omega$ be the map from $B$ onto $T$ defined above. Now a relation $R\left(x_{B}, y_{B}, z_{B}\right)=1$ holds in $B$ if and only if

$$
R\left(x_{B}, y_{B}, z_{B}\right)(i)=1
$$

for each $i \in P_{2}$. Similarly a relation $S(X, Y, Z)=1$ holds in $T$ if and only if

$$
S(X, Y, Z)(i)=1
$$

for each $i \in N$, that is if and only if the relation

$$
S\left(x, y, y^{20 t} z y^{-20 t}\right)=1
$$

holds in $A$ for each $i \in N$. Now $A=A / D_{1}$, so that $S\left(x, y, y^{20 t} z y^{-20 i}\right)=1$ in $A$ if and only if the relation

$$
S\left(x_{A}, y_{A}, y_{A}^{20 i} z_{A} y_{A}^{-20 i}\right)(t)=1
$$

holds in $S$ for all but finitely many $t \in P_{1}$. With this in mind it is not difficult to show that $\omega$ is a homomorphism. The fact that the kernel of $\omega$ is equal to $D_{2}$ may be proved in a similar way. However, the argument is rather more complicated, and it is necessary in the course of the proof to use the normal form theorem in $S$. We refer the reader to [3] for details. This shows that $\omega$ is an isomorphism between $B / D_{2}=\bar{B}$ and $T$, so we may identify $\bar{B}$ with $T$ under $\omega$. From now on, we shall make this identification without further comment. This completes the proof of Lemma 4.1.

We next consider $\bar{B}=\bar{B} \mid \bar{D}$, and, by abuse of notation, we use $x, y$ and $z$ to denote the images of $X, Y$ and $Z$ in $\overline{\bar{B}}$.

LeMMA 4.2. $E=\left\langle y, y^{3} z y^{-3}\right\rangle$ and $F=\left\langle x y, y^{3} z y^{-3}\right\rangle$ are both two-generator free groups. Let

$$
\begin{aligned}
& H=\left\langle y^{i}\left(y^{3} z y^{-3}\right) y^{-i}: i \in Z\right\rangle \leqq E, \\
& K=\left\langle(x y)^{i}\left(y^{3} z y^{-3}\right)(x y)^{-i}: i \in Z\right\rangle \leqq F,
\end{aligned}
$$

and let $\theta$ be the isomorphism between $H$ and $K$ defined by

$$
\theta\left(y^{i}\left(y^{3} z y^{-3}\right) y^{-i}\right)=(x y)^{i}\left(y^{3} z y^{-3}\right)(x y)^{-i}
$$

for each $i \in Z$. Then $\bar{B}=*(E, F ; H, K, \theta)$.

Proof. A word $w=w(x, y, z)$ is equal to 1 in $\overline{\bar{B}}$ if and only if the corresponding word $w_{B}=w(X, Y, Z)$ of $\bar{B}$ lies in $\bar{D}$. Hence $w=1$ in $\overline{\bar{B}}$ if and only if the value $w_{B}(i)$ of $w_{B}$ at $i$ is trivial for all but finitely many $i \in N$. The proof is 
now completed analogously with that of Lemma 3.1, using the normal form theorem in $A$.

We wish to prove that every ependomorphism of $B$ is an automorphism. Proceeding as in the proof of Proposition 3, we have shown that every ependomorphism of $B$ induces one of $\bar{B}$. We must now show that every ependomorphism of $\bar{B}$ induces one of $\bar{B}$. To this end we prove

LEMMA 4.3. $\overline{\bar{B}}$ is residually finite.

Proof. We wish to prove that given any non-trivial element $g$ of $\overline{\bar{B}}$ there is a normal subgroup $N_{g}$ of finite index in $\overline{\bar{B}}$ such that $g \notin N_{g}$. Suppose first that $g \notin H=K$. Now $H$ is normal in $\overline{\bar{B}}$ and $\overline{\bar{B}} / H$ is a free group of rank two, so the result follows, in this case, from the fact that free groups are residually finite. (See, e.g., [4], p. 144, problem 14.)

Now suppose that $g \in H$. Since $E$ is free, we may find a normal subgroup $M_{1}$ of finite index in $E$ such that $g \notin M_{1}$. Let $M_{2}$ be the image of $M_{1}$ under the map $\chi: E \rightarrow F$ given by

$$
\begin{aligned}
\chi\left(y^{3} z y^{-3}\right) & =y^{3} z y^{-3} \\
\chi(y) & =x y .
\end{aligned}
$$

Since the restriction of $\chi$ to $H$ is equal to the amalgamation map $\theta$, it follows that $g \notin M_{2}$. If $M$ is the normal closure in $\overline{\bar{B}}$ of the subgroup of $\overline{\bar{B}}$ generated by $M_{1}$ and $M_{2}$, then

$$
\overline{\bar{B}} / M \cong *\left(E / M_{1}, F / M_{2} ; H M_{1} / M_{1}, K M_{2} / M_{2}, \pi\right)
$$

where $\pi$ is the induced amalgamation map from $H M_{1} / M_{1}$ to $K M_{2} / M_{2}$. Thus $\overline{\bar{B}} / M$ is the generalised free product of two finite groups, and so there is a finite homomorphic image (the permutational product) of $\overline{\bar{B}} / M$ embedding $E / M_{1}$ and $F / M_{2}$. (See [5]). Since $g$ has non-trivial image in $E / M_{1}$, the result follows.

Lemma 4.4. $\bar{D}$ is the intersection of all the normal subgroups of finite index in $\bar{B}$.

Proof. Let $R$ be the intersection of the normal subgroups of finite index in $\bar{B}$. Since $\bar{B} / \bar{D}$ is residually finite, it follows that $R \leqq \bar{D}$. For each $i \in N$, we define $\bar{A}_{i}$ to be $\{f \in \bar{C}: f(j)=1$ for all $j \neq i\}$. Then for each $i \in N$, we have $A_{i} \leqq \bar{D}$ and $\bar{A}_{i} \cong A$. If $Q$ is any normal subgroup of finite index in $\bar{B}$, then $Q \cap A_{i}$ is of finite index in $A_{i}$. However, it is easy to prove that for any integer $t$, the adjunction of the relation $y^{t}=1$ to the relations of $\bar{A}$ causes $\bar{A}$ to collapse onto the identity. Consequently $\bar{A}$ has no normal subgroups of finite index. Thus $Q \cap \bar{A}_{i}=A_{i}$ for each $i \in N$ and so $\bar{D} \leqq Q$. This is true for each such $Q$, and so $\bar{D} \leqq R$. Thus $\bar{D}=R$, as required. 
Now $\bar{B}$ is finitely generated, and so it follows from Lemma 4.4 that $\phi(\bar{D})=\bar{D}$ for every ependomorphism $\phi$ of $\bar{B}$. We now find the ependomorphisms of $\overline{\bar{B}}$ and see which of these are induced from ependomorphisms of $\bar{B}$. Finally, we investigate which of these are induced from ependomorphisms of $B$.

LEMMA 4.5. Every ependomorphism $\psi$ of $\overline{\bar{B}}$ may be written, modulo an inner automorphism, in one of the following forms, where $\varepsilon_{i}= \pm 1$ for each $i=1,2,3$, and $k, r$ and $s$ are integers.

(i) $\psi(y)=y^{e_{1}}\left(y^{k} z y^{-k}\right)^{s} x^{r}, \psi(x)=x^{\varepsilon_{2}}, \psi\left(y^{3} z y^{-3}\right)=\left(y^{k} z y^{-k}\right)^{\varepsilon_{3}}$

(ii) $\psi(y)=y^{\varepsilon_{1}}\left(y^{k} z y^{-k}\right)^{s} x^{r}, \psi(x)=\left(y^{k} z y^{-k}\right)^{\varepsilon_{2}}, \psi\left(y^{3} z y^{-3}\right)=x^{\varepsilon_{3}}$.

Proof. Since $\overline{\bar{B}}$ is finitely generated and residually finite it is Hopf. (See, e.g., [4], p. 415.) Thus every ependomorphism of $\overline{\bar{B}}$ is an automorphism. Further, it is easy to check from the defining relations of $\overline{\bar{B}}$ that

$$
x\left(y^{i} z y^{-i}\right) x^{-1}=y^{i} z y^{-i} \quad \text { for each } i \in Z .
$$

Consequently $\psi(x)$ commutes with $\psi\left(y^{i} z y^{-i}\right)$ for each $i \in Z$, and we may use Theorem 1 to investigate the normal form structure of $\psi(x), \psi(y)$ and $\psi(z)$. Suppose first that one of $\psi(x)$ and $\psi\left(y^{3} z y^{-3}\right)$ lies in a conjugate of a factor, but not in a conjugate of $H$. It follows, as in the proof of Lemma 3.2, that

$$
\psi\left(\left[y^{3} z y^{-3}, y^{4} z y^{-4}\right]\right)=1 \text {. }
$$

But

$$
\left[y^{3} z y^{-3}, y^{4} z y^{-4}\right] \neq 1,
$$

so this contradicts the fact that $\psi$ is an automorphism of $\overline{\bar{B}}$. Next suppose that $\psi(x)$ has cyclically reduced length greater than one. Without loss of generality, we may assume that $\psi(x)$ is cyclically reduced, and then, by a length argument, we see that every element of $\overline{\bar{B}}$ which commutes with $\psi(x)$ is also cyclically reduced. Now $x$ commutes with every element of $H$, that is, with every element of $\left\langle y^{3} z y^{-3}\right\rangle^{\overline{\bar{B}}}$, the normal closure of $y^{3} z y^{-3}$ in $\overline{\bar{B}}$. Thus $\psi(x)$ commutes with every element of $\left\langle\psi\left(y^{3} z y^{-3}\right)\right\rangle^{\overline{\bar{B}}}$. But if $\psi\left(y^{3} z y^{-3}\right)$ does not lie in a conjugate of $H$, then its normal closure contains some elements which are not cyclically reduced and which therefore cannot commute with $\psi(x)$. Consequently $\psi\left(y^{3} z y^{-3}\right)$ lies in a conjugate of $H$. A similar argument deals with the case in which $\psi\left(y^{3} z y^{-3}\right)$ has cyclically reduced length greater than one. Consequently one of $\psi(x)$ and $\psi\left(y^{3} z y^{-3}\right)$ lies in a conjugate of $H$.

We will deal with the case in which $\psi\left(y^{3} z y^{-3}\right)$ lies in a conjugate of $H$. The other case may be dealt with in a similar way. Since $H$ is normal in $\overline{\bar{B}}$, we must have $\psi\left(y^{3} z y^{-3}\right)=h$ for some $h \in H$. Further $\overline{\bar{B}} / H$ is a free group of rank two 
generated by the images of $x$ and $y$ under the canonical map $\overline{\bar{B}} \rightarrow \overline{\bar{B}} / H$, and $\psi$ induces a map $\psi_{1}$ of $\overline{\bar{B}} / H$ onto $\overline{\bar{B}} /\langle h\rangle^{\overline{\bar{B}}}$. Since free groups are Hopf (see, e.g., [4], Theorem 2.13, p. 109) it follows that $\langle h\rangle^{\overline{\bar{B}}}=H$, and that $\psi_{1}$ is an automorphism of $\overline{\bar{B}} / H$. Thus Theorem 2 may be applied to $\overline{\bar{B}} / H$ and its automorphism $\psi_{1}$. We wish to find the total exponent to which $y$ appears in $\psi(x)$. We write $\psi(x)$ in normal form

$$
\psi(x)=h_{1} y^{\alpha_{1}}(x y)^{\beta_{1}} y^{\alpha_{2}}(x y)^{\beta_{2}} \cdots y^{\alpha_{n}}(x y)^{\beta_{n}}
$$

where $h_{1} \in H$ and $\alpha_{i}, \beta_{i} \in Z$ for each $i=1,2, \cdots, n$, and we use the fact that $\psi(x)$ commutes with every element of $\langle h\rangle^{\overline{\bar{B}}}=H$, and the relations of $\overline{\bar{B}}$ to deduce that

$$
\alpha_{1}+\beta_{1}+\alpha_{2}+\beta_{2}+\cdots+\alpha_{n}+\beta_{n}=0
$$

and that $h_{1}=1$. Theorem 2 now shows that there exist elements $g \varepsilon\langle x, y\rangle, h^{\prime} \in H$ and integers $s, t, \varepsilon_{1}= \pm 1$ and $\varepsilon_{2}= \pm 1$, such that

$$
\begin{aligned}
& \psi(x)=g x^{\varepsilon_{2}} g^{-1} \\
& \psi(y)=g x^{s} y^{\varepsilon_{1}} x^{t} g^{-1} h^{\prime} .
\end{aligned}
$$

Now $H$ is normal in $\overline{\bar{B}}$, so we may conjugate by $g x^{s}$ and obtain $\psi$, modulo an inner automorphism, in the form

$$
\psi\left(y^{3} z y^{-3}\right)=h_{2}, \psi(x)=x^{\varepsilon_{2}}, \psi(y)=y^{\varepsilon_{1}} x^{r} h_{3}
$$

for some elements $h_{2}, h_{3} \in H$, and some integers $r, \varepsilon_{1}= \pm 1$ and $\varepsilon_{2}= \pm 1$. We now consider $\overline{\bar{B}} /\langle x\rangle^{\overline{\bar{B}}}$. By checking the defining relations of $\overline{\bar{B}}$, we find that this is the free group of rank two generated by the images of $y$ and $z$ under the canonical map $\overline{\bar{B}} \rightarrow \overline{\bar{B}} /\langle x\rangle^{\overline{\bar{B}}}$. Moreover, $\psi$ induces an automorphism of $\overline{\bar{B}} \mid\langle x\rangle^{\overline{\bar{B}}}$, so Theorem 2 may again be applied. Since $y$ appears to total exponent zero in any generator of $H$, we deduce that there is an element $g_{1}$ of $E$ and integers $s$ and $\varepsilon_{3}= \pm 1$ such that

$$
h_{2}=g_{1}\left(y^{3} z y^{-3}\right)^{\varepsilon_{3}} g_{1}^{-1}, y^{\varepsilon_{1}} h_{3}=g_{1}\left(y^{e_{1}}\left(y^{3} z y^{-3}\right)^{5}\right) g_{1}^{-1} \text {. }
$$

But $g_{1}=h_{4} y^{l}$ for some $h_{4} \in H$ and some integer $l$, so we see that, modulo an inner automorphism by $h_{4}, \psi$ takes the form

$$
\psi(x)=x^{\varepsilon_{2}}, \psi(y)=y^{\varepsilon_{1}}\left(y^{k} z y^{-k}\right)^{s} x^{r}, \psi\left(y^{3} z y^{-3}\right)=\left(y^{k} z y^{-k}\right)^{\ell_{3}}
$$

for some integers $k, r, s, \varepsilon_{1}= \pm 1, \varepsilon_{2}= \pm 1$ and $\varepsilon_{3}= \pm 1$. A similar proof deals with the case where $\psi(x)$ lies in a conjugate of $H$, and this yields an automorphism of type (ii).

To prove that $B$ is Hopf, it is sufficient to show that for every ependomorphism $\theta$ of $B$, some power of $\theta$ is an automorphism. Now if $\theta$ induces $\bar{\theta}$ on $\bar{B}$ and $\overline{\bar{\theta}}$ on $\overline{\bar{B}}$, then $\theta^{i}$ induces $\bar{\theta}^{i}$ on $\bar{B}$ and $\overline{\bar{\theta}}^{i}$ on $\overline{\bar{B}}$. But Lemma 4.5 shows that modulo 
an inner automorphism of $\overline{\bar{B}}$, for every ependomorphism $\psi$ of $\overline{\bar{B}}$, some power of $\psi$ will take the form (i) as above with $\varepsilon_{1}=\varepsilon_{2}=\varepsilon_{3}=1$. Moreover, if

$$
\psi(y)=y\left(y^{k} z y^{-k}\right)^{s} x^{r}, \psi(x)=x, \psi\left(y^{3} z y^{-3}\right)=y^{k} y^{-k},
$$

then, modulo an inner automorphism,

$$
\psi^{2}(y)=y\left(y^{2 k-3} z y^{-(2 k-3)}\right)^{2 s} x^{2 r}, \psi^{2}(x)=x, \psi^{2}\left(y^{3} z y^{-3}\right)=y^{2 k-3} z y^{-(2 k-3)}
$$

This allows us to make certain restrictions on $r, s$ and $k$. We now sketch a proof of

LEMMA 4.6. If $\bar{\theta}$ is an ependomorphism of $\bar{B}$, and $\bar{\theta}$ induces $\overline{\bar{\theta}}$ on $\overline{\bar{B}}$, where $\overline{\bar{\theta}}$ takes the form (i) above, with $\varepsilon_{1}=\varepsilon_{2}=\varepsilon_{3}=1$, then $r=s=0, k \geqq 3$ and $k \equiv 3(\bmod .20)$.

Proof. We may write $\bar{\theta}$ in the form

$$
\bar{\theta}(\boldsymbol{Y})=\boldsymbol{Y}\left(\boldsymbol{Y}^{k} \boldsymbol{Z} \boldsymbol{Y}^{-k}\right)^{s} \boldsymbol{X}^{\boldsymbol{r}} \boldsymbol{\xi}, \bar{\theta}(\boldsymbol{X})=\boldsymbol{X} \eta, \bar{\theta}\left(\boldsymbol{Y}^{3} \boldsymbol{Z} \boldsymbol{Y}^{-3}\right)=\boldsymbol{Y}^{k} \boldsymbol{Z} \boldsymbol{Y}^{-k} \zeta,
$$

where $\zeta, \eta$ and $\zeta$ all lie in $\bar{D}$. Let $n$ be the smallest integer such that $\xi(i)=\eta(i)$ $=\zeta(i)=1$ for all $i \geqq n$. Now the relations of $A$ show that if $i \geqq-20$, or if $i<-20$ and $i$ is congruent to $0,1,2,3,4,5$ or 6 modulo 20 , then

$$
\left[Y^{i}\left(Y^{3} Z Y^{-3}\right) Y^{-i}, X\right](t)=1 \quad \text { for all } t \in N .
$$

Thus for each of these values of $i$

$$
\left[Y^{i}\left(Y^{3} Z Y^{-3}\right) Y^{-i}, X\right]=1
$$

is a relation of $\bar{B}$.

Suppose that $r \neq 0$. By considering, if necessary, $\bar{\theta}^{2}$ in place of $\bar{\theta}$, we may assume that $|r|>1$. We apply $\bar{\theta}$ to the above relations, and evaluate the expression

$$
\bar{\theta}\left(\left[\boldsymbol{Y}^{i}\left(\boldsymbol{Y}^{3} Z \boldsymbol{Y}^{-3}\right) \boldsymbol{Y}^{-i}, \boldsymbol{X}\right]\right)(t)
$$

at a suitably chosen $t \in N$. Using the relations of $A$, we find that if $|r|>1$, then

$$
\ddot{\theta}\left(\left[\boldsymbol{Y}^{i}\left(\boldsymbol{Y}^{3} \boldsymbol{Z} \boldsymbol{Y}^{-3}\right) \boldsymbol{Y}^{-i}, \boldsymbol{X}\right]\right)(t) \neq 1
$$

for any $t>n$ such that $20 t+k>3$, and for any $i \leqq-(20 t+k-2)$. This contradiction shows that we must have $r=0$.

Next we consider the value of $k$. We have proved that $r=0$. This property of $r$ is preserved by taking powers of $\bar{\theta}$, so we may again consider powers of $\bar{\theta}$ in place of $\bar{\theta}$. We have already shown that

$$
\bar{\theta}\left(\left[Y^{-(20 t+k-2)}\left(Y^{3} Z Y^{-3}\right) Y^{20 t+k-2}, X\right]\right)(t) \neq 1
$$

whenever $t>n$ and $20 t+k>3$. Thus 


$$
\bar{\theta}\left(\left[Y^{-(20 t+k-2)}\left(Y^{3} Z Y^{-3}\right) Y^{20 t+k-2}, X\right]\right) \neq 1 .
$$

Consequently $-(k-2)$ is not congruent to $0,1,2,3,4,5$ or 6 modulo 20 . Thus $k \equiv l(\bmod .20)$ where $3 \leqq l \leqq 15$. Let $\pi$ be the map $\pi: Z \rightarrow Z$ defined by

$$
\pi(i)=2 i-3 \text {. }
$$

Then, by considering powers of $\bar{\theta}$ in place of $\bar{\theta}$, we see that for every $j \in N$ the congruence class of $\pi^{j}(k)$ modulo 20 must lie between 3 and 15 . This shows that $k \equiv 3,8$ or 13 (mod. 20), so replacing $\bar{\theta}$ by a suitable power of itself, we may assume that $k \equiv 3$ (mod. 20). We observe that this property of $k$ is invariant under the operation of taking powers of $\bar{\theta}$.

Next we consider $s$. If $s \neq 0$, then, as before, we may assume that $|s|>1$ and that $s \equiv 0$ or $1(\bmod .3)$. Since $k \equiv 3(\bmod .20)$ we may put $k=20 m+3$, where $m \in Z$. We consider the expressions

$$
\bar{\theta}\left(\left[Y^{-(20(t+m)+14)}\left(Y^{3} Z Y^{-3}\right) Y^{20(t+m)+14}, X\right]\right)(t)
$$

and

$$
\bar{\theta}\left(\left[Y^{-(20(t+m)+15)}\left(Y^{3} Z Y^{-3}\right) Y^{20(t+m)+15}, X\right]\right)(t)
$$

where again $t>n$ and $20 t+k>3$. Since $-14 \equiv 6(\bmod .20)$ and $-15 \equiv 5$ (mod. 20) these expressions both represent the trivial element of $\bar{A}$. But consideration of the relations of $\bar{A}$ shows that for $|s|>1$ and $s \equiv 0$ or 1 (mod. 3) these two relations of $\bar{A}$ are incompatible. (See [3] for details.) Thus $s=0$, as required.

Finally we prove that $k \geqq 3$. Suppose not. Then since $k \equiv 3(\bmod .20)$ we may write $k$ in the form $k=-20 m+3$ where $m>0$. Let

$$
c_{r}=\left[X^{-1},\left(Y^{-(20 r+10)} Z Y^{20 r+10}\right)^{-1}\right]
$$

Then the relations of $A$ show that

$$
c_{r}(t)= \begin{cases}1 & \text { if } t \neq r \\ y^{-1} & \text { if } t=r\end{cases}
$$

However $\langle y\rangle^{\bar{A}}=\bar{A}$ and so we must have $\left\langle c_{r}\right\rangle^{B}=\bar{A}_{r}$. We now evaluate $\bar{\theta}\left(c_{r}\right)(t)$ for each $t \in N$, and discover that for $t>n$,

$$
\bar{\theta}\left(c_{r}\right)(t)= \begin{cases}1 & \text { if } t \neq r+m \\ y^{-1} & \text { if } t=r+m\end{cases}
$$

We choose $r$ such that $r+m>n$ and consider $\bar{\theta}\left(\bar{A}_{r}\right)=\left\langle\bar{\theta}\left(c_{r}\right)\right\rangle^{B}$. If $\bar{\theta}\left(c_{r}\right)(t) \neq 1$ for some $t \leqq n$, then, since $\bar{B} \geqq \bar{D}$ and since $\bar{A}$ is centreless, we must have

$$
\bar{\theta}\left(\bar{A}_{\mathrm{r}}\right) \cap \bar{A}_{\mathrm{t}} \neq\langle 1\rangle
$$

for that value of $t$. Thus $\bar{A}_{r+m}$ is a proper homomorphic image of $\bar{A}_{r}$. But this 
contradicts the fact that $\bar{A}$ is Hopf, and so we must have $\bar{\theta}\left(\bar{A}_{r}\right)=\bar{A}_{r+m}$ for each $r \in N$ such that $r+m>n$. But $\bar{\theta}(\bar{D})=\bar{D}$, and so it follows that $m<n$ and

$$
\bar{\theta}\left(\bar{A}_{1} \times \bar{A}_{2} \times \cdots \times \bar{A}_{n-m}\right)=\bar{A}_{1} \times \bar{A}_{2} \times \cdots \times \bar{A}_{n} .
$$

Let $i$ be any integer such that $1 \leqq i \leqq n-m$ and consider $\bar{\theta}\left(\bar{A}_{i}\right)=\langle\bar{\theta}(c)\rangle^{B}$. Just as before, we see that if

$$
\left\langle\bar{\theta}\left(c_{i}\right)(j)\right\rangle^{\bar{A}}=\bar{A}
$$

for any $j$ such that $1 \leqq j \leqq n$, then $\bar{\theta}\left(c_{i}\right)(k)=1$ for all $k \neq j$. Since $m>0$, it follows that there is some $k(1 \leqq k \leqq n)$ such that $\bar{A}_{k} \neq \bar{\theta}\left(\bar{A}_{j}\right)$ for any $j$ such that $1 \leqq j \leqq n-m$. Thus $\bar{A}_{k}$ is generated by $t(t \geqq 2)$ proper normal subgroups $N_{1}, N_{2}, \cdots N_{t}$, where these subgroups commute element-wise. It is clear that at least one of $N_{1}, N_{2}, \cdots N_{t}$, say $N_{1}$, contains a cyclically reduced element $c$ which does not lie in the amalgamated subgroup $H_{k}$ of $\bar{A}_{k}$. Suppose $c$ lies in a factor of $\overline{A_{k}}$. Let $d \notin H_{k}$ be an element of the other factor. Then, since $N_{1}$ is normal in $\bar{A}_{k}$, we have $d c d^{-1} c^{-1} \in N_{1}$, so that $N_{1}$ contains a cyclically reduced element of length greater than one. Thus in all cases $N_{1}$ contains a cyclically reduced element of length greater than one, and so it also contains elements which are not cyclically reduced, but which have cyclically reduced length greater than one. But the elements of $N_{i}(2 \leqq i \leqq t)$ all commute with the elements of $N_{1}$. Thus, by a length argument, we see that all the subgroups $N_{i}(2 \leqq i \leqq t)$ lie within $H_{k}$, so that $N_{1}$ and $H_{k}$ together generate $\bar{A}_{k}$. It follows that $N_{1}$ contains an element, $c$ say, which lies in a factor. But the elements of $N_{i}(2 \leqq i \leqq t)$ all commute with $c$ and lie in $H_{k}$. Since the factors are free, we deduce that $\bar{A}_{k} / N_{1}$ is cyclic. But it is easy to prove, from the defining relations of $\bar{A}$, that $\bar{A}$ is perfect. Thus $N_{1}=\bar{A}_{k}$, contradicting the fact that $N_{1}$ is a proper normal subgroup of $\bar{A}_{k}$. Consequently we must have $m \leqq 0$, and so $k \geqq 3$ as required.

Next we investigate which of these ependomorphisms $\bar{\theta}$ of $\bar{B}$ can be induced from an ependomorphism $\theta$ of $B$. We have

$$
\bar{\theta}(\boldsymbol{Y})=\boldsymbol{Y} \xi, \bar{\theta}(\boldsymbol{X})=\boldsymbol{X} \eta, \bar{\theta}\left(\boldsymbol{Y}^{3} Z \boldsymbol{Y}^{-3}\right)=\boldsymbol{Y}^{20 m+3} Z \boldsymbol{Y}^{-(20 m+3)} \zeta
$$

for some $m>0$, and some elements $\xi, \eta, \zeta$ of $\bar{D}$. Recalling the identification of $\bar{B}$ with $T \leqq \bar{C}$ given in Lemma 4.1 , we prove

LEMMA 4.7. Let $\theta$ be an ependomorphism of $B$, inducing an ependomorphism $\bar{\theta}$ of $\bar{B}$, where $\bar{\theta}$ takes the above form. Let $n$ be the smallest integer such that $\xi, \eta$ and $\zeta$ all satisfy

$$
\xi(i)=\eta(i)=\zeta(i)=1
$$

for all $i>n$. Then $m=0$, and $\theta$ induces on $\Pi_{1 \leqq i \leqq n} \bar{A}_{i}$ an automorphism $\bar{\theta}_{n}$ with the following structure:

There is a permutation $\pi$ of the set $\{1,2, \cdots, n\}$ such that for each $i,(1 \leqq i \leqq n)$, 


$$
\bar{\theta}_{n}\left(\bar{A}_{i}\right)=\bar{A}_{\pi(i)}
$$

and if $\bar{A}_{i}, \bar{A}_{\pi(i)}$ are identified with $\bar{A}$ in the obvious way, then $\bar{\theta}_{n}$ induces an inner automorphism of $\bar{A}$.

PROOF. We write $\theta$ in the form

$$
\theta\left(y_{B}\right)=y_{B} \xi \xi_{1}, \theta\left(x_{B}\right)=x_{B} \eta \eta_{1}, \theta\left(z_{B}\right)=y_{B}^{20 m} z_{B} y_{B}^{-20 m} \zeta \zeta_{1}
$$

where $\xi_{1}, \eta_{1}, \zeta_{1} \in D_{2}$ and $\xi, \eta, \zeta$ are elements of $B$ such that

$$
\xi\left(2^{k}+i\right)=\eta\left(2^{k}+i\right)=\zeta\left(2^{k}+i\right)=1
$$

whenever $2^{k}+i \in P_{2}$ and $i>n$. Let $r \in P_{2}$ be chosen in such a way that

$$
\xi_{1}(i)=\eta_{1}(i)=\zeta_{1}(i)=1
$$

whenever $i>r$. Suppose $m \neq 0$. Then by Lemma 4.6, $m>0$ and so we may choose $k>r$ in such a way that $k \neq 2^{s}+i$ for any $s>0$ and any $i$ such that $0 \leqq i \leqq n$, and such that $k+m=2^{t}$ for some $t>0$. Now the relations of $S$ show that

$$
\left[y_{B}^{-10} z_{B} y_{B}^{10}, x_{B}\right](i)=1
$$

for all $i \in P_{2}$. It follows that

$$
\left[y_{B}^{-10} z_{B} y_{B}^{10}, x_{B}\right]=1
$$

is a relation of $B$. However it is easy to check from the relations of $S$, that when $k$ is chosen as above we have

$$
\theta\left(\left[y_{B}^{-10} z_{B} y_{B}^{10}, x_{B}\right]\right)(k) \neq 1 .
$$

This contradiction shows that we must have $m=0$.

To prove the remaining part of Lemma 4.7 , we return to the group $\bar{B}$ and its ependomorphism $\bar{\theta}$. The proof is then completed in a manner entirely analogous to the proof that $k \geqq 3$ in Lemma 4.6 , so details are omitted here. The fact that every ependomorphism of $A$ is an inner automorphism (Lemma 3.2) completes the proof.

We may now complete the proof of Proposition 4. It is sufficient to prove that for every ependomorphism $\theta$ of $B$, some power of $\theta$ is an automorphism. Consequently, working modulo an inner automorphism, and using Lemmas 4.5, 4.6 and 4.7 , we may assume that $\theta$ takes the form

$$
\theta\left(y_{B}\right)=y_{B} \xi \xi_{1}, \theta\left(x_{B}\right)=x_{B} \eta \eta_{1}, \theta\left(z_{B}\right)=z_{B} \zeta \zeta_{1}
$$

where $\xi_{1}, \eta_{1}, \zeta_{1} \in D_{2}$, and $r \in P_{2}$ is chosen in such a way that

$$
\xi_{1}(i)=\eta_{1}(i)=\zeta_{1}(i)=1
$$

whenever $i>r$, and where $\xi, \eta, \zeta$ are elements of $B$ such that 


$$
\xi\left(2^{k}+i\right)=\eta\left(2^{k}+i\right)=\zeta\left(2^{k}+i\right)=1
$$

whenever $2^{k}+i \in P_{2}$ and $i>n$, where $n$ is defined as in Lemma 4.7. We consider the element

$$
w_{t}=\left[y_{B}^{-20 t-11} z_{B} y_{B}^{20 t+11}, x_{B}\right]
$$

of $B$. The relations of $S$ show that

$$
w_{t}(k)= \begin{cases}1 & \text { if } k>t \\ p q^{-1} p^{-1} & \text { if } k=t .\end{cases}
$$

Now let $k$ be chosen in such a way that $k \in P_{2}, k>\max (t, r)$ and $k \neq 2^{s}+i$ for any $s>0$ and any $i$ such that $1 \leqq i \leqq n$. It is easy to check from the relations of $S$ that we then have $\theta\left(w_{t}\right)(k)=1$. Moreover, if $t>r$ and $t \neq 2^{s}+i$ for any $s>0$ and any $i$ such that $1 \leqq i \leqq n$, we have $\theta\left(w_{t}\right)(t) \neq 1$. Now $S$ is simple, and so we have

$$
S=\left\langle w_{t}(t)\right\rangle^{S}
$$

for each $t \in P_{2}$. But the groups $S_{i}$ are exactly the minimal normal subgroups of $B$, so for each $i \in P_{2}$ we have either $\theta\left(S_{i}\right)=S_{j}$ for some $j \in P_{2}$ or $\theta\left(S_{i}\right)=1$. It follows that if $t>r$, and $t \neq 2^{s}+i$ for any $s>0$ and for any $i$ such that $1 \leqq i \leqq n$, then we must have $\theta\left(S_{t}\right)=S_{t}$.

We now consider those $t \in P_{2}$ which take the form $2^{s}+i$ with $s>0$ and $1 \leqq i \leqq n$. Let $x_{B, i}, y_{B, i}$ and $z_{B, i}$ be elements of $B$ defined in the following way:

$$
\boldsymbol{x}_{B, i}(k)=\boldsymbol{y}_{B, i}(k)=z_{B, i}(k)=1
$$

if $k \in P_{2}$ and $k \neq 2^{s}+i$ for any $s>0$, and for each $s>0$

$$
\begin{aligned}
& x_{B, i}\left(2^{s}+i\right)=b^{-1} a \\
& y_{B, i}\left(2^{s}+i\right)=p \\
& z_{B, i}\left(2^{s}+i\right)=p^{20 \cdot 2 s} a p^{-20 \cdot 2 s+1} .
\end{aligned}
$$

The form of $\bar{\theta}_{n}$ shows that there are elements $g_{i}$ of $B$ and $\xi^{(i)}, \eta^{(i)}$ and $\zeta^{(i)}$ of $D_{2}$ such that for each $i(1 \leqq i \leqq n)$,

$$
\begin{aligned}
& \theta\left(x_{B, i}\right)=g_{i} x_{B, \pi(i)} g_{i}^{-1} \xi^{(i)} \\
& \theta\left(y_{B, i}\right)=g_{i} y_{B, \pi(i)} g_{i}^{-1} \eta^{(i)} \\
& \theta\left(z_{B, i}\right)=g_{i} z_{B, \pi(i)} g_{i}^{-1} \xi^{(i)} .
\end{aligned}
$$

We choose $r^{\prime} \in P_{2}$ such that for each $i(1 \leqq i \leqq n)$

$$
\xi^{(i)}(t)=\eta^{(i)}(t)=\zeta^{(i)}(t)=1
$$

for all $t \in P_{2}$ such that $t>r^{\prime}$. By considering the element 


$$
w_{t}^{\prime}=\left[y_{B, i}^{-20 t-11} z_{B, i} y_{B, i}^{20 t+11}, x_{B, i}\right]
$$

of $B$ (where $t \in P_{1}$ ), and using the same argument as before, we prove that if $k>r^{\prime}$ and $k=2^{s}+i$ for some $s>0$ and some $i$ such that $1 \leqq i \leqq n$, then we must have

$$
\theta\left(S_{k}\right)=S_{2^{s+\pi(i)}}
$$

Let $r^{\prime \prime}=\max \left(r, r^{\prime}\right)$. It follows from the above reasoning that

and that

$$
\operatorname{ker}(\theta) \leqq S_{3} \times S_{5} \times \cdots \times S_{r^{*}}
$$

$$
\theta\left(S_{3} \times S_{5} \times \cdots \times S_{r^{\prime \prime}}\right)=S_{3} \times S_{5} \times \cdots \times S_{r^{\prime \prime}}
$$

But the groups $S_{i}$ are all simple. Consequently $\operatorname{ker}(\theta)=1$, and so $\theta$ is an automorphism of $B$. Thus $B$ is Hopf, as required.

This completes the proof of Theoerem A.

\section{The proof of Theorem B}

We now use a similar method to construct a finitely generated group $G_{0}$ isomorphic to its own direct square. For this construction we shall need the structure known as a Higman-Neumann-Neumann (HNN) or Britton extension, and the associated normal form theorem, Britton's lemma. Details of the theorems required here may be found in [3], Chapter 1.

We begin by constructing the 'base group' $S_{0}$. We take $S$ ' to be the group $S_{\rho, \sigma \tau}$ where $\rho, \sigma$ and $\tau$ are defined on the negative integers as follows:

Let $\Delta_{t}=\{n \in Z:-20 t \leqq n \leqq-20 t+19\}$. Then $\rho, \sigma$ and $\tau$ fix each $\Delta_{t}$ set-wise, and induce on $\Delta_{t}$ permutations $\rho_{t}, \sigma_{t}$ and $\tau_{t}$ defined (as products of disjoint cycles) by

$$
\begin{aligned}
\rho_{t}= & (-20 t+18,-20 t+19) \\
\sigma_{t}= & (-20 t+9,-20 t+10)(-20 t+12,-20 t+15,-20 t+13) \\
& (-20 t+14,-20 t+19) \\
\tau_{t}= & (-20 t+12,-20 t+13)(-20 t+14,-20 t+19,-20 t+18) .
\end{aligned}
$$

The subgroup $\langle a, p\rangle$ of $S^{\prime}$ is free, and so we may make an HNN extension $S^{\prime \prime}$ of $S^{\prime}$ by adding to $S^{\prime}$ the generator $c$ and the relations

$$
\begin{aligned}
& c^{-1} a c=a \\
& c^{-1} p c=p^{2} .
\end{aligned}
$$

An application of Britton's lemma shows that the subgroup $\left\langle b^{-1} a, c\right\rangle$ of $S^{\prime \prime}$ is free. Let $H$ be a group with presentation

$$
H=\left\langle d, e, f: e d e^{-1}=d^{2}, d f d^{-1}=f^{2}\right\rangle .
$$


$H$ is the result of two HNN extensions, first of the free group $\langle f\rangle$ by the generator $d$, according to the relation $d f d^{-1}=f^{2}$, and then of the resulting group by the generator $e$ according to the relation $e d e^{-1}=d^{2}$. It follows from Britton's lemma that the subgroups $\langle e\rangle$ and $\langle f\rangle$ of $H$ generate their free product, which is a free group of rank two. We may, therefore, form the free product of $S^{\prime \prime}$ and $H$ amalgamating the subgroups $\left\langle b^{-1} a, c\right\rangle$ of $S^{\prime \prime}$ and $\langle e, f\rangle$ of $H$ according to the relations

$$
\begin{aligned}
b^{-1} a & =e \\
c & =f .
\end{aligned}
$$

The resulting group is

$$
\begin{aligned}
S_{0}=\left\langle a, p, b, q, c, d: a^{i} p^{\rho(i)}\right. & =b^{\tau(i)} q^{\sigma \tau(i)} \text { for each } i \in I, c^{-1} a c=a, \\
c^{-1} p c & \left.=p^{2},\left(b^{-1} a\right) d\left(b^{-1} a\right)^{-1}=d^{2}, d c d^{-1}=c^{2}\right\rangle .
\end{aligned}
$$

Let $C$ be the cartesian power $C=S_{0}^{N}$, and let $G_{0}$ be the subgroup of $C$ generated by

$$
\begin{aligned}
x & =\left(b^{-1} a, b^{-1} a, b^{-1} a, \cdots\right) \\
y & =(p, p, p, \cdots) \\
z & =\left(p^{10} a p^{-9}, p^{20} a p^{-19}, p^{30} a p^{-29}, \cdots\right) \\
t & =(c, c, c, \cdots) \\
u & =(d, d, d, \cdots) .
\end{aligned}
$$

We define $P_{1}$ to be the subset of $N$ consisting of all odd numbers, $P_{2}$ to be the corresponding set of even numbers, and $C_{1}, C_{2}$ to be the cartesian powers $S_{0}^{P_{1}}, S_{0}^{P_{2}}$ respectively, considered in the natural way as subgroups of $C$. For each $f \in G_{0}$, we define functions $f_{1}, f_{2} \in C$ as follows:

For each $j=1,2$

$$
f_{j}(i)= \begin{cases}f(i) & \text { if } i \in P_{j} \\ 1 & \text { otherwise }\end{cases}
$$

We define

$$
G_{j}=\left\{f_{j}: f \in G_{0}\right\}
$$

for each $j=1,2$. We prove that $G_{2} \leqq G_{0}$, from which it follows that $G_{0}=G_{1} \times G_{2}$.

Using the definitions of $\rho, \sigma$ and $\tau$ given above, we see that for each $t \in N$ the following relations hold in $S_{0}$ :

(1) $\left(b^{-1} a\right)\left(p^{20 t} a p^{-20 t+1}\right)\left(a^{-1} b\right)=p^{20 t} a p^{-20 t+1}$

(2) $\left(b^{-1} a\right)\left(p^{20 t-10} a p^{-20 t+11}\right)\left(a^{-1} b\right)=p^{20 t-9} a^{2} p^{-20 t+11}$ 
(3) $\left(b^{-1} a\right)\left(p^{20 t-13} a p^{-20 t+14}\right)\left(a^{-1} b\right)=p^{20 t-15} a^{-4} p^{-20 t+11} q$

(4) $\left(b^{-1} a\right)\left(p^{20 t-16} a p^{-20 t+17}\right)\left(a^{-1} b\right)=p^{20 t-16} a^{-4} p^{-20 t+12} q^{7} p^{-1} q^{-1}$.

Consider the element $\left[x^{-1}, z^{-1}\right]$ of $G_{0}$. By (1) and (2) we have

$$
\begin{aligned}
{\left[x^{-1}, z^{-1}\right] } & =\left(p^{11} a p^{-10}, 1, p^{31} a p^{-30}, 1, p^{51} a p^{-50}, 1, \cdots\right) \\
& =y z_{1} y^{-1} .
\end{aligned}
$$

Thus $G_{0}$ contains $z_{1}$ and all its conjugates by powers of $y$. It now follows, in a similar way, from (3) and (4) that $G_{0}$ contains $x_{1} y_{1}$ and $y_{1}$. Consequently $G_{0}$ also contains $x_{2}, y_{2}$ and $z_{2}$. Further,

$$
\begin{aligned}
\boldsymbol{u}^{-1} \boldsymbol{x}_{2} \boldsymbol{u} & =\left(1, d^{-1}\left(b^{-1} a\right) d, 1, d^{-1}\left(b^{-1} a\right) d, 1, \cdots\right) \\
& =\left(1, d\left(b^{-1} a\right), 1, d\left(b^{-1} a\right), 1, \cdots\right) \\
& =u_{2} x_{2}
\end{aligned}
$$

which shows that $G_{0}$ also contains $u_{2}$. Also

$$
\begin{aligned}
t^{-1} u_{2} t & =\left(1, c^{-1} d c, 1, c^{-1} d c, 1, \cdots\right) \\
& =(1, c d, 1, c d, 1, \cdots) \\
& =t_{2} u_{2}
\end{aligned}
$$

which shows that $G_{0}$ contains $t_{2}$. Thus $G_{2}=\left\langle x_{2}, y_{2}, z_{2}, t_{2}, u_{2}\right\rangle \leqq G_{0}$, and so $G_{0}=G_{1} \times G_{2}$.

Let $\alpha: C \rightarrow C$ be the monomorphism defined by

$$
(f \alpha)(i)= \begin{cases}f(i-1) & \text { for } i>1 \\ 1 & \text { for } i=1\end{cases}
$$

Then $\left.\alpha\right|_{G_{1}}$ is an isomorphism of $G_{1}$ onto $G_{2}$, so in order to complete the proof that $G_{0} \cong G_{0} \times G_{0}$, we need only show that $G_{2} \cong G_{0}$. To this end, we let $\beta: C \rightarrow C$ be the monomorphism defined by

$$
\begin{aligned}
(f \beta)(2 i) & =f(i) \\
(f \beta)(2 i-1) & =1 .
\end{aligned}
$$

We investigate the image of $G_{0}$ under $\beta$. Since

$$
\begin{aligned}
\beta(z) & =\left(1, p^{10} a p^{-9}, 1, p^{20} a p^{-19}, 1, \cdots\right) \\
& =t_{2} z_{2} y_{2}^{-1} t_{2}^{-1} y_{2}
\end{aligned}
$$

we see that $\beta$ maps $G_{0}$ onto $G_{2}$. Thus $\left.\beta\right|_{G_{1}}$ is an isomorphism between $G_{0}$ and $G_{2}$, so that $G_{1} \cong G_{2} \cong G_{0}$, as required. This completes the proof of Theorem $B$. 


\section{References}

[1] I. M. S. Dey and Hanna Neumann, 'The Hopf Property of Free Products', Math. Z. 117, (1970), 325-339.

[2] R. Camm, 'Simple Free Products', J. London Math. Soc. 28, (1953), 66-76.

[3] J. M. Tyrer, 'On Direct Products and the Hopf Property', D. Phil. Thesis, University of Oxford, (1971).

[4] W. Magnus, A. Karrass and D. Solitar, Combinatorial Group Theory, (Pure and Applied Mathematics, Vol. 13, Interscience Publishers, 1966).

[5] B. H. Neumann, 'An Essay on Free Products of Groups with Amalgamations', Philos, Trans. Roy. Soc. London, Ser. A. 246 (1954), 503-554.

New Hall

Cambridge

England 